\title{
Guest reviewers: volume 10
}

Correspondence: sckong@ied.edu.hk Department of Mathematics and Information Technology, The Hong Kong Institute of Education, 10 Lo Ping Road, Tai Po, New Territories, Hong Kong

\section{Contributing reviewers \\ The Editors express their thanks to the persons named below for contributing to the review process in respect of paper submissions received in 2015.}

Sergei Abramovich
Yacine Atif
Nada Bajnaid
Rachid Benlamri
Zane Berge
Jawad Berri
Lars Bollen
Bodong Chen
Chih-Ming Chen
Jacky Chen
Juanjuan Chen
Yang-Hsueh Chen
Zhi-Hong Chen
Gary Cheng
Hercy Cheng
Chih-Yueh Chou
Doris Choy
Jean-Noel Colin
Adam Cooper
Chung Wai Han
Jianye Hao
Shinobu Hasegawa
Yusuke Hayashi
Tsukasa Hirashima
Choi Wa Dora Ho
Wing Kei Ho
Jia-Fei Hong
Huei-Tse Hou

Cheng-Hsu Huang

Hsu-Wen Huang

Hing Keung Hung

Noriyuki Iwane

Sridhar Iyer

Morris S. Y. Jong

Yih-Ruey Juang

Akihiro Kashihara

Beaumie Kim

Fanny Klett

Joyce Koh

Kazuaki Kojima

Tatsuhiro Konishi

Hidenobu Kunichika

Ping Wai Kwok

Theresa Kwong

Chih-Hung Richard Lai

Lydia Lau

Nguyen-Thinh Le

Chi Fai Leung

Kui Chiu Issic Leung

Ping Li

Calvin C. Y. Liao

Chia-Ching Lin

Tzu-Chiang Lin

Yu-Tzu Lin

Chen-Chung Liu

Hsueh-jui Sarah Liu
Jon Mason

Noriyuki Matsuda

Kenji Matsuura

Moffat Matthews

Sahana Murthy

Kiyoshi Nakabayashi

Niels Pinkwart

Mercedes Rodrigo

Hitomi Saito

Kazuhisa Seta

Christian M. Stracke

Bernardo Tabuenca

Ryo Takaoka

Shikui Tu

Noriko Uosaki

Hao-Chuan Wang

Amali Weerasinghe

Eva Wong

Su Luan Wong

Tak Lam Ivan Wong

Chun-Ping Wu

Longkai Wu

Syo Yamamoto

Jane Yau

Fu-Yun Yu

Yuen Tak Yu

Joerg Zumbach 